\title{
Medizin und Recht: Therapieverzicht am Lebensende durch niedergelassene Ärzte
}

\author{
Rogler, Gerhard ; Mausbach, Julian
}

\begin{abstract}
The question, how and when an individual decision for and end of medical treatment or abdication of therapy should be made remains difficult. Ethical considerations have to be taken into account with respect to a humane and dignified decease. The goal of our manuscript there for is to achieve more certainty with respect to juridical and legal preconditions and frameworks. The conditions in Switzerland support general practitioners and family doctors to fulfill patients' requests, wishes and wills in an outpatient setting. An awareness and sensitization for a dignified and humane care at the end of life - despite social changes and an increased importance of health economics - as well increasing support of outpatient palliative care become more and more important. Existing legal certainty and security of practitioners, therefore, will be outlined in the following.
\end{abstract}

DOI: https://doi.org/10.1024/1661-8157/a001513

Other titles: Medicine and Law: abdication of therapy by practitioners at the end of life

Posted at the Zurich Open Repository and Archive, University of Zurich

ZORA URL: https://doi.org/10.5167/uzh-92816

Journal Article

Accepted Version

Originally published at:

Rogler, Gerhard; Mausbach, Julian (2013). Medizin und Recht: Therapieverzicht am Lebensende durch niedergelassene Ärzte. Praxis, 103(1):19-26.

DOI: https://doi.org/10.1024/1661-8157/a001513 


\section{Medizin und Recht: Therapieverzicht am Lebensende durch niedergelassene Ärzte}

Prof. Dr. med. Dr. phil. Gerhard Rogler und Dr. iur. Julian Mausbach RA

\section{Zusammenfassung}

Die Frage, wie die individuelle Grenzziehung zum Behandlungsabbruch oder Therapieverzicht aus ethischer Sicht vorzunehmen ist und wann diese Schritte möglicherweise mit Blick auf ein menschenwürdiges Sterben geboten sind, bleibt schwierig. Ziel des vorliegenden Beitrages ist es, zu erreichen, dass die Beantwortung dieser Frage nicht von einer Unsicherheit über die rechtlichen Voraussetzungen belastet ist. Es soll dargestellt werden, dass der Wunsch des Patienten auch und gerade durch Hausärzte und niedergelassene Mediziner in einem auf ambulante Behandlung und Betreuung ausgerichteten Setting umgesetzt werden kann. Die Sensibilisierung für eine würdevolle Gestaltung des Lebensendes, trotz oder angesichts eines gesellschaftlichen Wandels und einer Ökonomisierung der Medizin, sowie die weitere Stärkung ambulanter palliativer Angebote sind angezeigt. Die bestehende Rechtssicherheit soll daher vermittelt und dargestellt werden.

\section{Einleitung}

Wenn ein 45-jähriger Patient, der an der degenerativen neurologischen Erkrankung amyotropher Lateralsklerose (ALS) leidet und einen stark progredienten klinischen Verlauf zeigt, trotz künstlicher Ernährung und einer nicht-invasiven Masken-Überdruckbeatmung einen Punkt erreicht, an er seine Lebensqualität als nicht mehr ausreichend wahrnimmt, wie kann mit diesem Problem umgegangen werden? Wenn kein Lebenswille mehr besteht, weil Leben mit massivem Leiden verbunden ist, wie soll man mit Komplikationen der Erkrankung umgehen? Wenn sich im Verlauf eine Aspirationspneumonie, eine Lungenentzündung durch eingeatmetes Sekret einstellt, soll die Entzündung therapiert werden? Wenn sich die Lungenentzündung verschlechtert und eine zunehmende Atemnot eintritt, müssen hier Reanimationsmassnahmen eingeleitet und das Leben weiter erhalten werden? Wie ist die Situation zu werten, wenn in der Akutsituation einer schweren Atemnot ein Reanimation durchgeführt wurde, aber im Rahmen dieser Massnahmen ein hypoxischer Hirnschaden, eine Unterversorgung des Gehirns mit Sauerstoff mit der Folge einer irreversiblen Hirnschädigung entstanden ist? Wenn nun die Frau des Patienten als Betreuerin verlangt, dass die künstliche Beatmung oder die Intensivtherapie bei einem solchen Patienten beendet wird, wie kann damit umgegangen werden? Kann einem solchen Patienten iatrogen Leid zugefügt werden, um das Leben unter allen Umständen zu erhalten, sind hier Schmerzen für den Patienten weiterhin zu vertreten, hat die Lebenserhaltung hier Vorrang vor dem Patienten/Betreuer-Wunsch und dem Gebot der Schmerz- bzw. Leidenslinderung? 
So schwierig diese und ähnliche Fallkonstellationen bereits durch die Bedingungen des jeweiligen Einzelfalls sind, werden sie auch durch allgemeine Entwicklungen in ihrer Komplexität gesteigert. Die Kenntnis der Lebensumstände eines solchen Patienten oder einer solchen Patientin, die Entwicklung der subjektiven Einstellungen zur Erkrankung, der persönliche Blick auf die Lebensqualität können helfen, den Patientenwillen zu ermitteln. Diesen Einblick und diese Kenntnisse hat der niedergelassene Hausarzt, der einen solchen Patienten oder eine solche Patientin behandelt in weit grösserem Masse als der Spitalarzt, der den Patienten oder die Patientin vielleicht zum ersten Mal sieht. Auch in entsprechenden Spezialambulanzen wechseln die zuständigen Ärztinnen und Ärzte häufig. Daher kommt hier den hausärztlich Tätigen ein hohes Mass an Verantwortung und eine Aufgabe zu, die im Grunde nur von Ihnen erfüllt werden kann. Trotz der Bedeutung dieser Einsicht in Grundeinstellung, Lebenseinstellung, Krankheitseinstellung wird diese Verantwortung jedoch sehr häufig abgegeben. Es ist festzustellen, dass der Sterbeort sich immer mehr weg von zuhause verlagert. Dies ist angesichts der in Spitälern und Heimen angebotenen Betreuung und Behandlungsoptionen von Personen am Lebensende eine teilweise nachvollziehbare Entwicklung.

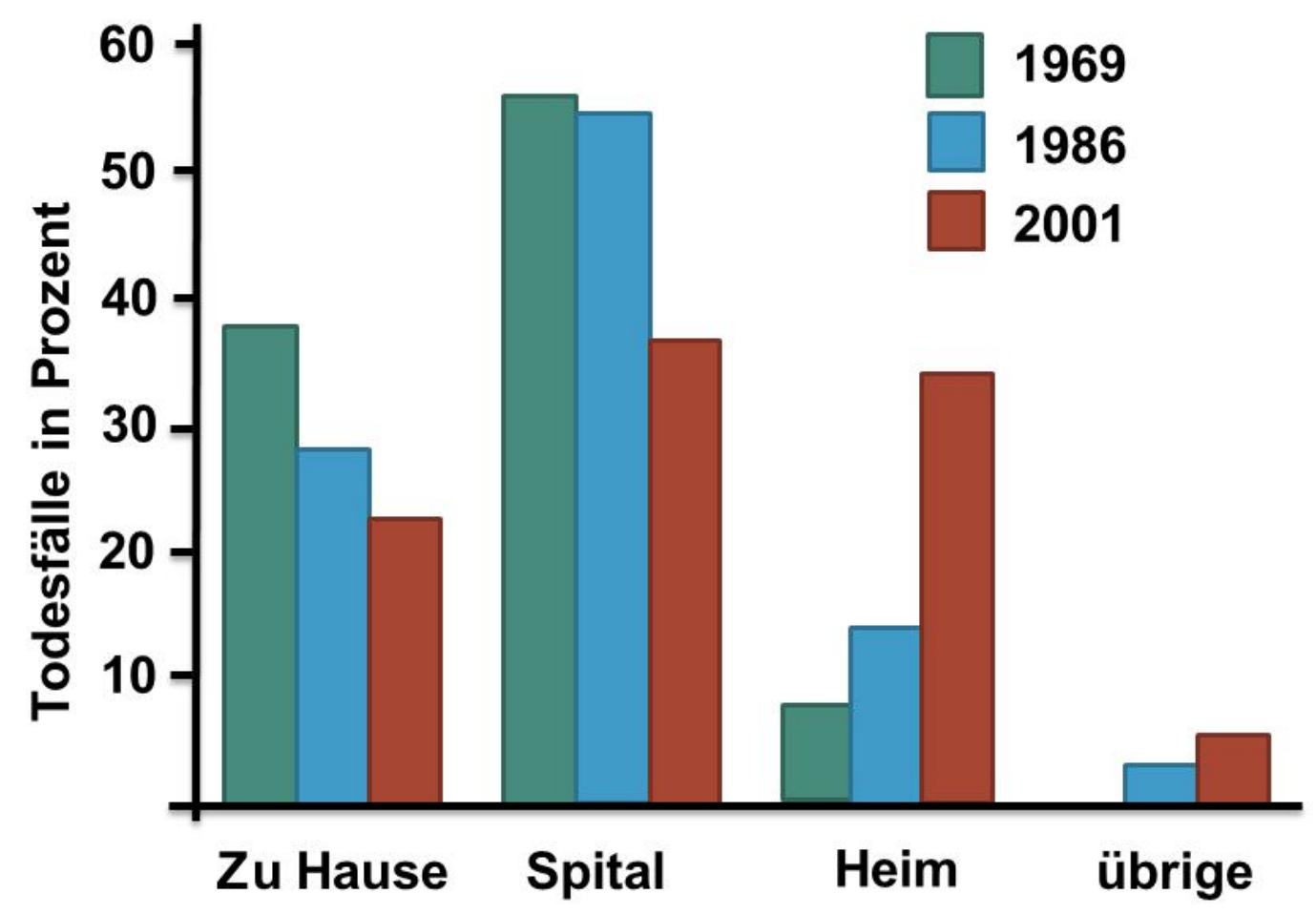

Abbildung 1: Anteil der Todesfälle nach Sterbeort in der Schweiz für die Jahre 1969, 1986 und 2001. Quelle: Susanne Fischer, Entscheidungsmacht und Handlungskontrolle am Lebensende, Eine Untersuchung bei Schweizer Ärztinnen und Ärzten zum Informations-und Sterbehilfeverfahren, Verlag für Sozialwissenschaften, Wiesbaden, Deutschland, 2008

Dieser Entwicklung steht aber in nicht wenigen Fällen der Wunsch der Patientinnen und Patienten gegenüber, in einer vertrauten Umgebung aus dem Leben scheiden zu dürfen. Um diesem Ansinnen gerecht werden zu können, stellen sich auch für niedergelassene Ärztinnen und Ärzte wichtige Fragen, die Unsicherheit erzeugen, wie im persönlichen Gespräch immer wieder bestätigt wird: Ist eine "Nicht-Einweisung" ins Spital bereits eine Form von Therapieverzicht oder unterlassener Hilfeleistung? Welche rechtlichen Voraussetzungen sind bei einem Therapieverzicht am Lebensende zu beachten? Wie kann ein solcher Schritt ambulant optimal vollzogen werden? 
Beeinflusst wird die Beantwortung zum einen durch den Ausbau der medizinische Behandlungs- und Therapiemöglichkeiten sowie den Fortschritt der Medizintechnik in den letzten Jahren. Aufgrund dieses Fortschritts bietet sich ein immer breiteres Spektrum an Massnahmen- und Behandlungsoptionen am Lebensende. Gerade die Intensivmedizin konnte von den teils enormen technischen Entwicklungen profitieren und ist heute mehr denn je in der Lage den Eintritt des Todes zu verzögern. Diese - an sich sehr zu begrüssenden und erfreulichen - Entwicklungen erschweren die Beantwortung der oben aufgeworfenen Fragen. So besteht zum einen weitgehend Einigkeit, dass nicht das technisch Mögliche allein die Grenzen einer Behandlung bestimmen kann und darf. Hocheffektive Rettungs- und Reanimationsmassnahmen sowie sonstige Vorgehensweisen, die es gestatten auch einen recht schlechten gesundheitlichen Allgemeinzustand relativ langfristig aufrechterhalten zu können, schaffen allerdings auch einen Anreiz, anstehende medizinisch-ethische Entscheidung herauszuschieben, zu verzögern oder, beispielsweise durch Überweisung eines bisher ambulant betreuten Patienten ins Spital, an andere Personen zu delegieren. Auch im Spital werden dann solche Entscheidungen oft nicht getroffen, man zieht sich darauf zurück, der Erkrankung ihren „natürlichen Verlauf unter Therapie“ (ein Widerspruch in sich) zu lassen.

Zum anderen wird durch die Entwicklung der modernen Medizin aber auch ein immer höherer Spezialisierungsgrad der einzelnen Mediziner erreicht. Dieser grundsätzlich zu begrüssende, weil den Erkenntnisgewinn der Medizin repräsentierender Fakt, kann als Kehrseite der Spezialisierung allerdings für eine vertrauensvolle Arzt-Patienten-Beziehung hinderlich sein. Deren Ausbildung ist schon durch die faktischen Umstände (beispielsweise dadurch, dass die Spezialisten die Patienten erst nach einer Vielzahl vorausgegangener anderer Arzt-Patienten-Kontakte begegnen) strukturell schwächer, als jene zu einem vertrauten niedergelassenen (Haus-)Arzt, der seine Patienten häufiger sieht und der über einen langen Zeitraum immer wieder konsultiert wird. Gerade eine vertrauensvolle Beziehung ist für den Umgang mit Fragen am Ende des Lebens jedoch ein nicht zu unterschätzender Vorteil und mithin eine wichtige Ressource für die Suche nach der Antwort auf die Frage, wie mit dem Wunsch der Patienten und Patientinnen umzugehen ist, in vertrauter Umgebung aus dem Leben scheiden zu dürfen.

Hinzu tritt, dass manche aktuelle Entwicklung im Gesundheitswesen mindestens gegenwärtig die Bildung einer vertrauensvollen Beziehung zwischen Arzt und Patient erschweren kann. Das Aufstellen von Leistungskatalogen, die Bezahlung der Spitäler nach Fallpauschalen (DRG-System) und die Definition von Guidelines und fast-track Prozeduren, mag eine ökonomisch angezeigte Entwicklung im derzeitigen gesellschaftlichen Gesamtgefüge sein. Sie bedingt allerdings, dass der Arzt im öffentlichen Verständnis mehr und mehr als Dienstleister erscheint, der auf Wünsche und Bedürfnisse zu reagieren hat und lediglich ein Angebot von "Gesundheitsleistungen" generieren soll. Eine Kenntnis des sozialen Hintergrunds eines Patienten ist jedoch essentiell, um eine adäquate palliative Therapie zu ermöglichen. Das „in Beziehung treten“ von Arzt und Patient, das langsam aufzubauende Vertrauensverhältnis als Grundlage vieler klinisch-ethischer Ansätze, hat unter diesen Umständen eine geringere Bedeutung. Eine Beratung in Kenntnis der spezifischen Situation des Betroffenen wird jedenfalls nicht erleichtert.

Erschwert wird die Entscheidungsfindung zudem, wenn wie oben dargestellt hinsichtlich der rechtlichen Rahmenbedingungen und Handlungs-Konsequenzen Unklarheiten und Unsicherheiten herrschen. Ziel des vorliegenden Beitrages ist es daher zu erreichen, dass die Beantwortung der Frage: Wie ist die komplexe und schwierige individuelle Grenzziehung zum Behandlungsabbruch oder Therapieverzicht aus ethischer Sicht vorzunehmen und wann sind diese Schritte möglicherweise mit 
Blick auf ein menschenwürdiges Sterben geboten, nicht von einer Unsicherheit über die rechtlichen Voraussetzungen belastet sind. Darüber hinaus soll dargestellt werden, dass der Wunsch des Patienten auch und gerade durch Hausärzte und niedergelassene Mediziner in einem auf ambulante Behandlung und Betreuung ausgerichteten Setting umgesetzt werden kann.

\section{Rechtslage am Lebensende}

Die einschlägigen Fallkonstellationen werden häufig unter Begriffen diskutiert, die eine grosse Nähe zum Strafrecht aufweisen. "Sterbehilfe" und "Suizidbeihilfe" sind dabei wohl die prominentesten. Dies ist nach Auffassung der Autoren für die Beantwortung der aufgeworfenen Fragen nicht immer hilfreich. Insbesondere da es vielmehr um die Frage geht, welche Vorgehensweise einzuhalten ist, damit eine dem Patientenwillen entsprechende Entscheidung umgesetzt werden kann. Gerade das am Patientenwillen ausgerichtete Vorgehen ist und soll im Bereich des Therapieverzichts, -abbruchs oder der Behandlungsbegrenzung nicht mit Strafe bewehrt sein. ${ }^{1}$ Die Fragen sind daher vielmehr mit Blick auf die Begriffe "Würde", "Selbstbestimmung" sowie "Nutzen“ und "Wirksamkeit" von Therapien zu beantworten. Auch die massgeblichen Richtlinien der Schweizerischen Akademie für Medizinische Wissenschaften (SAMW) richten ihren Fokus auf diese letztgenannten Begriffe und binden die Frage des Behandlungsabbruchs und -verzichts in eine Auseinandersetzung mit diesen ein. Sie legen damit Empfehlungen vor, welche eine Orientierung zur Entscheidungsfindung ermöglichen.

Die Richtlinien sind dabei nicht nur als eine Empfehlung zu interpretieren, sondern entfalten, durch den Einbezug in die Standesordnung der FMH über den Anhang $\mathrm{I}^{2}$ auch für (niedergelassene) Ärztinnen und Ärzte berufs- und standesrechtliche Wirkung.

Die für Entscheidungen am Lebensende heranzuziehende Richtlinien ${ }^{3}$ sind folgende:

- Patientenverfügungen (2009)

- Reanimationsentscheidungen (2008)

- Palliative Care (2006)

- Behandlung und Betreuung von älteren, pflegebedürftigen Menschen (2004)

- Betreuung von Patientinnen und Patienten am Lebensende (2004)

- Behandlung und Betreuung von zerebral schwerst geschädigten Langzeitpatienten (2003)

- Grenzfragen der Intensivmedizin (1999)

Darüber hinaus sind auf gesetzlicher Ebene in verschiedenen kantonalen Gesetzen einschlägige Normen enthalten. Diese sind, auch wenn ihr Anwendungsbereich sich nicht auf niedergelassenen Ärztinnen und Ärzte erstreckt ${ }^{4}$, für die Entscheidungsfindung ebenfalls, mindestens im Sinne einer

1 Beispielsweise die SAMW-Richtlinie zur Betreuung von Patientinnen und Patienten am Lebensende nimmt in ihrem Abschnitt zum Behandlungsverzicht und -abbruch unter Ziffer 3.2. an, dass ein solches Vorgehen „geboten“ sein kann; abrufbar unter http://www.samw.ch/de/Ethik/Richtlinien/Aktuell-gueltigeRichtlinien.html (zuletzt aufgerufen am 16.07.2013).

2 Anhang 1: Von der Ärztekammer übernommene Richtlinien der Schweizerischen Akademie der Medizinischen Wissenschaften und Ethische Grundsätze des Weltärztebundes; abrufbar unter http://www.fmh.ch/fmh/rechtliche_grundlagen/standesordnung.html (zuletzt aufgerufen am 16.07.2013) abrufbar.

3 Die Richtlinien der SAMW sind unter http://www.samw.ch/de/Ethik/Richtlinien/Aktuell-gueltigeRichtlinien.html (zuletzt aufgerufen am 16.07.2013) abrufbar.

4 Siehe hierzu § 1 Patientinnen- und Patientengesetz des Kantons Zürich (vom 5. April 2004, Ordnungsnummer 813.13); abrufbar unter: http://www.zhlex.zh.ch/Erlass.html?Open\&Ordnr=813.13. 
Orientierungshilfe, heranzuziehen. Für den Kanton Zürich lautet die entscheidende Norm des Patientinnen- und Patientengeset ${ }^{5}$ wie folgt:

$\S 31$. Bei tödlich erkrankten, nicht urteilsfähigen Patientinnen und Patienten können die Ärztinnen und Ärzte die kurative Behandlung einschränken oder einstellen, wenn

a. das Grundleiden mit aussichtsloser Prognose einen irreversiblen Verlauf genommen hat und

b. ein Hinausschieben des Todes für die Sterbenden eine nicht zumutbare Verlängerung des Leidens bedeutet und

c. der Verzicht auf eine Weiterführung der Behandlung dem mutmasslichen Willen der Patientinnen und Patienten entspricht.

2 Die Bezugspersonen oder die gesetzliche Vertretung sind von den behandelnden Ärztinnen und Ärzten für ihren Entscheid mit einzubeziehen. Bei unmündigen oder entmündigten Patientinnen und Patienten darf die Behandlung nicht gegen den Willen der gesetzlichen Vertretung eingeschränkt oder eingestellt werden.

3 Eine von der Patientin oder vom Patienten früher verfasste Verfügung bezüglich lebensverlängernder Massnahmen ist zu beachten. Sie ist unbeachtlich, wenn sie gegen die Rechtsordnung verstösst oder Anhaltspunkte dafür bestehen, dass die oder der Sterbende in der Zwischenzeit die Einstellung geändert hat.

Die Ebene, aus welcher sich nunmehr die eingangs dieses Abschnitts erwähnte enge begriffliche Nähe zur "Sterbehilfe" etc. ergibt, entspringt dem Strafgesetzbuch. ${ }^{6}$ Dies enthält einen Titel "Strafbare Handlungen gegen Leib und Leben“, in welchem sich die Straftatbestände zu den Tötungsdelikten in den Art. $111 \mathrm{ff}$. finden. Eben diese sind gegebenenfalls beim Versterben einer Person oder einem Suizidgeschehen einschlägig und unter Umständen auch beim Tod eines Patienten für die Beurteilung des Verhaltens des behandelnden Arztes massgeblich. Die verständliche Furcht vor strafrechtlichen Konsequenzen für ein bestimmtes ärztliches Vorgehen, macht es unumgänglich auch diese Ebene in die Bewertung von Therapieverzicht, -begrenzung und abbruch einzubeziehen. Die bereits hier zu treffende wichtigste Aussage zu dieser Ebene erscheint mit Schwarzenegger folgende: „Gar nicht in den Regelungsbereich des Strafrechts fallen[...] die medizinische Unterstützung und Betreuung von Sterbenden, soweit sie objektiv keine lebensverkürzende Wirkung haben (Sterbebegleitung). ${ }^{47}$

\section{Behandlungsabbruch und Therapieverzicht}

Unter einem Behandlungsabbruch versteht man das Nichtweiterführen, sei es durch Unterlassen der weiteren Behandlung oder durch deren aktiven Abbruch, einer begonnenen lebenserhaltenden Massnahme. In diesen Bereich fällt beispielsweise der Abbruch einer Beatmung des Patienten. $\mathrm{Er}$ wird vielfach unter die Kategorie der „passiven Sterbehilfe“ aber ebenso auch ${ }^{8}$ unter die Kategorie der "indirekten aktiven Sterbehilfe“ gefasst.

5 Patientinnen- und Patientengesetz des Kantons Zürich (vom 5. April 2004, Ordnungsnummer 813.13); abrufbar unter: http://www.zhlex.zh.ch/Erlass.html?Open\&Ordnr=813.13.

6 Schweizerisches Strafgesetzbuch vom 21. Dezember 1937 (SR. 311.0); abrufbar unter: http://www.admin.ch/ch/d/sr/c311_0.html.

7 Schwarzenegger Christian in: Niggli Wiprächtiger (Hrsg.), Basler Kommentar zum Strafrecht II, 3. Auflage 2013, Vor Art. 111, N 44.

8 Schwarzenegger, a.a.O., RN. 21. 
Unter einem Therapieverzicht wiederum versteht man das Nichtergreifen einer lebenserhaltenden Massnahme. Hierunter fällt etwa die Nichtaufnahme einer Reanimation. Diese wird üblicherweise als passive Sterbehilfe eingeordnet. Die Frage der Einordnung hat umfangreiche Diskussionen und Positionen hervorgebracht. ${ }^{9}$ Letztlich kommt es darauf aber mit Blick auf die Bewertung des Einzelfalles bei Patienten am Lebensende nicht mehr an. Dies hat etwa der deutsche Bundesgerichtshofes in seinem Urteil vom 25. Juni $2010^{10}$ wie folgt festgehalten: „Eine Differenzierung nach aktivem und passivem Handeln nach äußerlichen Kriterien [ist] nicht geeignet [...], sachgerecht und mit dem Anspruch auf Einzelfallgerechtigkeit die Grenzen zu bestimmen, innerhalb derer eine Rechtfertigung des Handelns durch den auf das Unterlassen oder den Abbruch der medizinischen Behandlung gerichteten Willen des Patienten anzuerkennen ist, müssen andere Kriterien gelten[...]." Die Entscheidungsfindung welche zu einem Therapieverzicht oder Behandlungsabbruch führt, „ist nur entsprechend dem erklärten oder mutmaßlichen Patientenwillen durch die Nichteinleitung oder den Abbruch lebensverlängernder Maßnahmen zulässig, um dem Sterben - ggf. unter wirksamer Schmerzmedikation - seinen natürlichen, der Würde des Menschen gemäßen Verlauf zu lassen“11.

Diese Einschätzung ist durch die Vorgaben zur Bewertung in der Schweiz bereits seit einiger Zeit vorgenommen worden. Dies verdeutlicht bereits ein Blick in die einschlägigen SAMW-Richtlinien, welche die Frage des Behandlungsverzichts und -abbruchs nicht trennen, sondern vielmehr unter einem Titel behandeln. ${ }^{12}$ Unabhängig davon, wie man ein unter die Begriffe des Therapieverzichts oder des Behandlungsabbruchs zu subsumierendes Vorgehens mithin rechtlich einordnen will, bedarf es der folgenden Voraussetzungen, damit es rechtmässig ist:

- Patientenwille:

Sofern der betroffene Patient urteilsfähig ist bedarf es seines erklärten Willens zum Abbruch der Behandlung bzw. zum Therapieverzicht. Dabei ist klar, dass die Kommunikation zwischen Arzt und Patient auf das jeweilige Individuum angepasst sein muss, um den entsprechenden Willen zu eruieren. Es kann hier kein neutrales Beratungsgespräch stattfinden. Die Diskussion der Wertigkeit einer Therapie muss auf den individuellen Hintergrund des Patienten abgestimmt werden. In einer Arzt - Patientenbeziehung sollte durch Kommunikation nach wie vor ein Vertrauensverhältnis aufgebaut werden. Wenn dieses Vertrauensverhältnis steht, kann ein Wunsch nach einem Therapieende oder passiver Sterbehilfe besser eingeschätzt werden. Gerade diese Voraussetzung kann in einer, idealerweise langjährig gewachsenen Beziehung zwischen einem niedergelassenen Arzt und seinen Patienten regelmässig gewährleistet werden. Dies wiederum ermöglicht die genaueste Eruierung des tatsächlichen Patientenwillens. Die Autonomie eines Patienten wird von verschiedenen Faktoren beeinflusst, etwa Alter und Bildungsniveau. So ist beispielsweise die Tendenz, Entscheidungen eines Arztes zu vertrauen, in Bevölkerungsschichten mit nichtakademischem Hintergrund stärker ausgeprägt. Da jedoch auch bei einem akademisch Gebildeten

9 Vgl. statt vieler Schwarzenegger, a.a.O., Vor Art. 111, N $48 \mathrm{ff}$.

10 http://juris.bundesgerichtshof.de/cgi-bin/rechtsprechung/document.py?Gericht=bgh\&Art=en\&Datum= Aktuell\&nr=52999\&linked=urt\&Blank=1\&file=dokument.pdf.

11 BGHSt 37, 376 (379).

12 Siehe sowohl die SAMW-Richtlinie Betreuung von Patientinnen und Patienten am Lebensende als auch jene zur Palliative Care, a.a.O. 
die Einblicke in die Hintergründe der Therapien, in Studiendaten und Primärdaten fehlen, kann diese Autonomie aber per se nie vollständig sein.

Sofern der Patient nicht (mehr) urteilsfähig ist, muss sein „mutmasslicher Wille" erforscht werden. Die Ermittlung des Patientenwillens kann dabei zunächst Patientenverfügung ${ }^{13}$ gestützt werden. Denn gemäss Art. 367 Zivilgesetzbuch $(Z G B)^{14}{ }^{\prime \prime}$,...] kann eine urteilsfähige Person [...] in einer Patientenverfügung festlegen, welchen medizinischen Massnahmen sie im Fall ihrer Urteilsunfähigkeit zustimmt oder nicht zustimmt.“

Vorausgesetzt ist dabei, dass die Patientenverfügung

- aktuell ist,

- valide Rückschlüsse auf den Willen des Patienten in der konkret gegebene Situation zulässt und des Weiteren,

- dass keine Anhaltspunkte für eine nachträgliche Änderung des in der Verfügung abgebildeten Willens vorliegen.

Auch hierbei kann ein Rückgriff auf ein gewachsenes Vertrauensverhältnis zwischen Patienten und Ärzten sehr hilfreich sein, um festzustellen, ob die Patientenverfügung in der konkreten Situation den genannten Anforderungen gerecht wird. Beispielsweise in den Fällen, in denen der Arzt gar als Berater bei der Erstellung der Patientenverfügung einbezogen wurde. Kann der Patientenwille nicht aufgrund einer Patientenverfügung zweifelsfrei festgestellt werden, so ist dieser durch den Einbezug der zur Vertretung berechtigten Person (in aller Regel die Angehörigen des betroffenen Patienten) ${ }^{15}$ zu ermitteln.

Erst wenn alle zugänglichen Möglichkeiten zur Erforschung des mutmasslichen Willens ausgeschöpft sind und die Vornahme dringlich ist, kann ein Handeln im wohlverstandenen Interesse (best interest) des Patienten erfolgen. Die SAMW Richtlinie zur Betreuung von Patientinnen und Patienten am Lebensende $^{16}$ versteht darunter „die Durchführung von medizinisch oder pflegerisch indiziert erscheinenden Massnahmen, denen ein hypothetischer vernünftiger Patient in der entsprechenden Situation voraussichtlich zustimmen würde." Dabei ist unter Berücksichtigung des Erwachsenenschutzrechts anzumerken, dass die Beurteilung, welche Massnahme vorzunehmen bzw. zu unterlassen ist, hinsichtlich der medizinischen Bewertung weiterhin in ärztlicher Kompetenz liegt, allerdings eine Erweiterung hinsichtlich der Information und Aufklärungspflichten erfolgt ist. Entsprechend Art. 377 ZGB ist ein Behandlungsplan aufzustellen, in welchem "die behandelnde Ärztin oder der behandelnde Arzt unter Beizug der zur Vertretung bei medizinischen Massnahmen berechtigten Person die erforderliche Behandlung [zu planen hat]".

Um diese gesetzeskonform vorzunehmen muss "die Ärztin oder der Arzt die vertretungsberechtigte Person über alle Umstände, die im Hinblick auf die vorgesehenen medizinischen Massnahmen wesentlich sind, insbesondere über deren Gründe, Zweck, Art, Modalitäten, Risiken, Nebenwirkungen und Kosten, über Folgen eines Unterlassens der Behandlung sowie über allfällige alternative Behandlungsmöglichkeiten [informieren]".

\footnotetext{
13 Auf die Möglichkeit eines Vorsorgeauftrages nach Art. 360 ff. ZGB wird hier aus Platzgründen nicht näher eingegangen.

14 Schweizerisches Zivilgesetzbuch vom 10. Dezember 1907 (Stand am 1. Januar 2013).

15 Siehe hierzu Art. 374 ZGB. Zur Reihenfolge der zur Vertretung Berechtigten siehe Art. 378 ZGB.

16 A.a.O.
} 
Ziel dieses Vorgehens ist es, die vertretungsberechtigte Person in die Lage zu versetzen, an Stelle und im Sinne der vertretenen Person, mithin nach deren mutmasslichen Willen zu entscheiden. Verdeutlicht wird dies dadurch, dass das Gesetz ausdrücklich vorsieht, dass „soweit möglich [...] auch die urteilsunfähige Person in die Entscheidfindung einbezogen [wird]". Damit wird deutlich herausgestellt welch hoher Stellenwert dem Willen der Patienten und der Ausübung des Selbstbestimmungsrechts eingeräumt wird.

\section{- Medizinische Faktoren}

Bei der Entscheidungsfindung ist der urteilsfähige Patient über die Prognose, den voraussichtlicher Behandlungserfolg im Sinne der Lebensqualität sowie die Belastung durch die vorgeschlagene Therapie soweit zu aufzuklären, dass sie ihre Entscheidung informiert treffen kann. Ist der betroffenen Patient urteilsunfähig, so sind diese Informationen entsprechend an die zur Vertretung berechtigte Person zu erteilen. Besonderes Augenmerk ist bei den vorliegenden Fragen dabei darauf zu richten, dass hier mit Blick auf die Lebensqualität nicht nur die allfällige Wirksamkeit einer Behandlung, sondern vor allem ihr möglicher Nutzen für den betroffenen Patienten thematisiert wird.

Ein typisches Beispiel hierfür mag der fortgeschrittene Krebs der Bauchspeicheldrüse, das metastasierte Pankreaskarzinom sein. Klinische Studien zeigen, dass der Nutzen einer aggressiven Chemotherapie in dieser Situation sehr limitiert ist. Sie führt in 90-95 von 100 Fällen zu einer Verschlechterung der Lebensqualität durch die mit ihr Verbundenen Nebenwirkungen (Übelkeit, Erbrechen usw) ohne das Leben zu verlängern. Sie kann durch die Nebenwirkungen in den allermeisten Fällen die Lebensqualität für die verbleibende Lebenszeit einschränken. Eine hochdosierte Schmerztherapie mit Morphinen hingegen wird in den allermeisten Fällen die Lebensqualität verbessern, kann aber bei einer unabsichtlichen Überdosierung durch den Patienten zum Tod führen. Gerade wenn aber kein Nutzen für den Patienten durch eine Therapie zu erwarten ist sollte deren Abbruch bzw. der Verzicht auf sie erwogen werden. Dies betrifft grundsätzlich auch wirksame Methoden.

Sind die zur Verfügung stehenden Methoden gar unwirksam oder unzweckmässig, so statuieren die Richtlinien der SAMW zur Betreuung von Patientinnen und Patienten am Lebensende, dass "die Respektierung des Patientenwillens [...] dann an ihre Grenzen [stösst], wenn ein Patient Massnahmen verlangt, die unwirksam oder unzweckmässig sind oder die mit der persönlichen Gewissenshaltung des Arztes, mit der ärztlichen Standesordnung oder dem geltenden Recht nicht vereinbar sind." Eine solche Therapie ist nicht fortzuführen bzw. nicht zu beginnen.

Mitunter mag die Einschätzung eines Patienten in der oben genannten recht aussichtslosen Situation aber auch dahin gehen, sich eine Therapie zu wünschen, deren Schädigungspotential hoch, deren potentieller Nutzen im Einzelfall ebenfalls hoch, dieser Einzelfall jedoch extrem selten ist. So könnten 1-2 von 100 Patienten von einer solchen Therapie profitieren, während sie 98 schadet. Sollte hier der unbedingte Therapiewunsch des Patienten berücksichtigt werden obwohl die Risk/Benefit Ratio hier u.U. bei 25:1 liegt?

Verneint man als betreuender Mediziner diese Frage, so kann man nicht zur Durchführung der Behandlung gezwungen werden. Es ist aber jedenfalls sicherzustellen, dass die medizinische Betreuung des Patienten fortgeführt wird. Sei dies in einem weiterhin kurativ ausgerichteten Ansatz oder in einem palliativ-medizinischen Setting. 
Der Abbruch oder die Begrenzung einer Therapie ist mithin möglich und geboten, wenn mindestens eine der folgenden Voraussetzungen gegeben ist ${ }^{17}$ :

1. Die Durchführung der Massnahme entspricht nicht dem Patientenwillen.

2. Nach Einschätzung der behandelnden Ärzte oder Ärztinnen liegt keine medizinische Indikation für die Massnahme vor.

\section{Palliative Massnahmen als begleitenden Massnahme}

Wird die Entscheidung zu einem Therapieverzicht getroffen, stellen sich weitere Fragen. Verdeutlicht sein dies an folgendem Beispiel. Zerebrale Metastasen bei einer Patientin mit Brustkrebs (Mammakarzinom), bei der die Prognose infaust ist, ziehen die Notwendigkeit einer palliativen Schmerzbehandlung nach sich. Die unmittelbare Frage, die letztlich auch den Bereich der indirekten aktiven Sterbehilfe betrifft, ist jene nach dem Primat der Schmerzbekämpfung zum Beispiel mit Morphinen. Ist es möglich, dieser Patientin ein Morphinpräparat in die Hand zu geben und ihr zu erklären, bis zu welcher Dosierung sie gehen kann, ohne dass eine Unterdrückung der Atemfunktion eintritt? Ihr gleichzeitig aber auch zu erläutern, ab welcher Dosierung, bei selbst dann nicht mehr erträglichen Schmerzen, eine Atemlähmung und somit der Tod eintreten kann? Die Frage ist also, ob die Schmerzbekämpfung mit Morphinen bis in einen Bereich fortgeführt werden kann, in dem die Atemdämpfung durch diese Medikamente möglicherweise schon selbst zum Tod führt. Auch bei einem Patienten mit fortgeschrittenem Krebs der Bauchspeicheldrüse (siehe oben) können diese Fragen entscheidend sein. Prinzipiell ist hier keine Heilung möglich. Durch Einwachsen des Tumors in die Nervengeflechte an der Hinterwand der Bauchhöhle entstehen stärkste Schmerzen. Diese sind häufig durch höchste Dosen von Schmerzmitteln nur inkomplett zu beseitigen. Darf diesem Patienten ein Morphinpräparat an die Hand gegeben werden, mit der gleichzeitigen Information wie viel Tabletten notwendig wären, um dem Leben ein Ende zu setzen? Es scheint evident zu sein, dass dies in einer Situation, in der sich Arzt und Patient als Kunde und Leistungsanbieter zum ersten Mal sehen, recht problematisch ist. Die Situation scheint anders zu sein, wenn zwischen Hausarzt und Patient ein ausgebildetes Vertrauensverhältnis besteht.

Aus juristischer Sicht ist klar, dass eine Behandlung die eine möglichst hohe Lebensqualität und eine würdevolle Gestaltung der Gesamtsituation anstrebt, rechtmässig durchgeführt werden kann. Dies gilt selbst dann, wenn der Einsatz dieser Massnahmen zur Verhinderung von Leiden und Schmerzen eine Reduktion der verbleibenden Lebensdauer mit sich führen könnte. Ein solches Setting wäre ggf. unter dem Begriff der sogenannten „aktiven indirekten Sterbehilfe“ zu fassen. Ein solches Vorgehen wird weder vom Strafgesetzbuch unter Strafe gestellt, noch wird es sonst von der Rechtsordnung missbilligt. Vielmehr erachten auch die massgeblichen Richtlinien der SAMW ein entsprechendes Vorgehen für zulässig. Es ist daher nicht ersichtlich, warum es nicht in einem ambulanten Setting, so es im Einzelfall die vorzugswürdige Massnahme ist, Anwendung finden sollte. Dabei kommt es darauf an, dass der eingeschlagenen Weg im geäusserten oder mutmasslichen Willen der betroffenen Patienten ist und von der Motivation getragen wird, ein würdevolles Sterben ohne Schmerzen zu

17 In diesem Sinne auch das Positionspapier „Therapiezieländerung und Therapiebegrenzung in der Intensivmedizin“ der Sektion Ethik der Deutschen interdisziplinären Vereinigung für Intensiv- und Notfallmedizin. Abrufbar unter: http://www.diviorg.de/fileadmin/pdfs/sektionen/Ethik/Positionspapier _Ethik_2012.pdf. 
erreichen. Primär ist ein solches Verhalten mithin nicht auf Lebensbeendigung oder -verkürzung gerichtet, selbst wenn diese aber in Kauf genommen wird oder werden muss.

Im Falle eines Therapieabbruchs bzw. einer Therapiereduktion ist eine weitergehende Begleitung im Sinne des Patienten mithin unerlässlich. Gerade hier steht die Frage, wie der Würde und dem Willen des Patienten bezüglich der verbleibenden Lebensdauer am wirkungsvollsten Rechnung getragen werden kann. Klar ist, dass eine optimale palliativmedizinische Betreuung in dem hierbei zu erstellenden Plan regelmässig als wichtiges Element fungieren muss.

Fraglich ist, wie und in welcher Form dies auch in einer vertrauten und gewohnten Umgebung realisiert werden kann. Die stationäre palliative Betreuung kann hier aus medizinischen Gründen geboten sein. Angesichts der Entwicklungsschritte, die im Bereich der Palliativmedizin gemacht wurden und die eine recht weitreichende Flexibilität gestatten, wie man den Ort der palliativen Betreuung bestimmen und ggf. verändern oder anpassen kann (Siehe etwa http://www.pallnetz.ch) sind aber immer auch mögliche ambulanten Massnahmen in Erwägung zu ziehen.

\section{Fazit}

Für den Hausarzt und die Hausärztin und den niedergelassen Arzt oder die niedergelassene Ärztin sind die Fragen des Therapieverzichts und des Behandlungsabbruches heute von zentraler Bedeutung. Insbesondere wenn es darum geht, dem Patientenwunsch nach einem Versterben in vertrauter Umgebung gerecht zu werden.

Die notwendige medizinische Begleitung und Betreuung sind durch sie im Team mit entsprechenden ambulanten Pflegeangeboten zu bewerkstelligen. Angesichts der demographischen Entwicklung in einer immer älter werdenden Gesellschaft steht zudem zu erwarten, dass die Fragen noch weiter an Bedeutung gewinnen werden. Die sich für den Hausarzt und Hausärztinnen und anderen niedergelassene Ärzte und Ärztinnen dabei ergebende Chance, aufgrund einer häufig grossen Nähe zum Patienten, den Patientenwillen hinsichtlich des Umgangs mit Therapieverzicht und Behandlungsabbruch nicht nur in der akuten Situation zu berücksichtigen, sondern diesen Willen bereits in der gewachsenen Arzt-Patientenbeziehung valide zu eruieren, stellt einen grossen Vorteil zur Durchsetzung und Beachtung des Selbstbestimmungsrechts des Patienten dar. Die wohl häufig anzunehmende Situation, dass der (noch) urteilsfähige Patient mit dem Arzt die anfallenden Fragen besprechen und klären, allenfalls gar diese Gespräche zur Anfertigung einer Patientenverfügung oder Beauftragung einer Person zur Übernahme der Personensorge im Falle der Urteilsunfähigkeit nutzen kann, gilt es stärker in die gesellschaftliche Aufmerksamkeit zu rücken.

Mit den im konkreten Fall eines Therapieverzichts oder Behandlungsabbruchs auftretenden rechtlichen Fragen kann der niedergelassene Arzt und die niedergelassene Ärztin dann mindestens ebenso gut zu umgehen, wie dies im bei einer stationären Versorgung der Fall ist. Dabei ist die Sorge sich selbst einem unkalkulierbarem juristischem Risiko auszusetzten nicht begründet. Vorausgesetzt, dass das medizinisch Notwendige im ambulanten Rahmen gewährleistet werden kann, ist es möglich einem bekannten und belegbaren Patientenwunsch nach vertrauter Umgebung am Lebensende nachzukommen.

Schwieriger ist dies, wenn eine Klärung des Patientenwillens nicht stattgefunden hat oder stattfinden konnte. Die hier auftretenden Schwierigkeiten zur Bewertung der Situation können im Umfeld eines Spitals, in welchem neben dem Ärzteteam auch auf ethische und rechtliche Bewertung geschulte 
Personen zurückgegriffen werden, gegebenenfalls besser gelöst werden. Dies allerdings als Standard anzuerkennen erscheint dann nicht sachgerecht, wenn der Wunsch des Patienten erkennbar nicht auf ein Versterben im Spital gerichtet war. In diesen Fällen wäre eine zukünftige Erweiterung von Angeboten, die es auch dem niedergelassenen Arzt und der niedergelassenen Ärztin ermöglichen, sich ethischen und rechtlichen Rat einzuholen wünschenswert. Selbstverständlich ist dabei, dies sei festgehalten, sicherzustellen, dass eine nur stationär zu gewährleistende Versorgung auch ebendort vorzunehmen ist.

Darüber hinaus gilt es darauf hinzuweisen, dass die vorliegend vorgestellten Fallbeispiele aus einer Fallgruppe stammen, die entsprechend der Auswertung der Ursachen für die Inanspruchnahme von organisierter Suizidbeihilfe in der Schweiz eine entscheidende Rolle spielen. So stehen Tumorerkrankungen an erster Stelle der Statistik und machen nahezu 50\% der Fälle von organisierten assistierten Suiziden aus und werden von den neurodegenerative Erkrankungen, wie amyotrophe Lateralsklerose und Morbus Parkinson, an zweiter Stelle gefolgt. ${ }^{18}$ Die hohe Inanspruchnahme von organisierter Suizidbeihilfe in diesen Erkrankungsfelder lässt vermuten, dass möglicherweise auch eine Unsicherheit hinsichtlich der juristischen Fragen auf Seiten der behandelnden Mediziner dazu führt, dass sich Patienten schliesslich organisierten assistierten Suiziden zuwenden. Der Aufarbeitung dieser Unsicherheiten und der vorhandenen Wissensdefizite diente dieser Artikel.

Die Frage, ob die Stärkung der ambulanten Strukturen mit einem stärkeren Einbezug der Hausärzte und Hausärztinnen oder der niedergelassenen Mediziner in die Entscheidungen am Lebensende, zu einer stärkeren Beachtung des vorhandenen Wunsches in vertrauter Umgebung aus dem Leben zu scheiden können und zu einem Einfluss auf die Inanspruchnahme organisierter Sterbehilfe führt, muss offen bleiben. Die weitere Sensibilisierung für eine würdevolle Gestaltung des Lebensendes, trotz oder angesichts eines gesellschaftlichen Wandels und einer Ökonomisierung der Medizin, sowie die weitere Stärkung ambulanter palliativer Angebote sind aber in jedem Falle angezeigt.

Wenn hierbei eine Unsicherheit in juristischen Fragen besteht, muss sie durch eine entsprechende Vermittlung von Wissen im Idealfall bereits im Medizinstudium oder durch zusätzliche Fort- und Weiterbildungskurse wie sie auch das Kompetenzzentrum Medizin - Ethik - Recht Helvetiae (MERH) der Universität Zürich anbietet, beseitigt werden.

Die bestehende Rechtssicherheit kann vermittelt und dargestellt werden. Wir hoffen, dazu beigetragen zu haben.

18 Siehe hierzu Bundesamt für Statistik Todesursachenstatistik2009 Sterbehilfe (assistierter Suizid) und Suizid in der Schweiz; abrufbar unter: http://issuu.com/sfso/docs/1257-

0900 ?mode=embed\&layout=http\%3A\%2F\%2Fskin.issuu.com\%2Fv\%2Flight\%2Flayout.xml\&showFlipBtn=tru e. 